\title{
Surveillance urinaire des professionnels de la santé exposés aux antinéoplasiques dans le cadre de leur travail : revue de la littérature de 2010 à 2015
}

\author{
Céline Poupeau, Christel Roland et Jean-François Bussières
}

\section{RÉSUMÉ}

Contexte : Il existe de plus en plus de données sur la présence de traces de médicaments dangereux dans l'urine des professionnels de la santé exposés à ces médicaments.

Objectif : Présenter une revue de la littérature scientifique concernant la surveillance urinaire de professionnels de la santé exposés aux antinéoplasiques dans le cadre de leur travail.

Sources de données : Recherche sur PubMed avec les Medical Subject Headings (MeSH) "occupational exposure " et "antineoplastic agents" ainsi que sur Google Scholar avec les termes " antineoplastic ", " urine » et "occupational exposure".

Sélection des études et extraction des données : L'examen a porté sur tous les articles en anglais et en français ayant trait aux professionnels de la santé exposés à des médicaments dangereux dans le cadre de leur travail, publiés entre le $1^{\text {er }}$ janvier 2010 et le 31 décembre 2015. Les articles ne comportant pas de résultats urinaires et ceux concernant les vétérinaires ainsi que les revues de littérature, les éditoriaux, les lettres à la rédaction et les résumés de congrès ont été exclus.

Synthèse des données : Vingt-quatre articles ont été retenus. Les études ont été menées dans 52 établissements de santé provenant de sept pays. Elles regroupaient 826 travailleurs exposés à des médicaments dangereux et 175 témoins, notamment des infirmiers ( $n=16$ études), des pharmaciens $(n=10)$, des assistants techniques en pharmacie $(n=8)$, des médecins $(n=7)$, des aides-soignants $(n=2)$ et autres $(n=8)$. Différentes méthodes analytiques ont été utilisées pour quantifier la présence de 13 médicaments dangereux, principalement le cyclophosphamide ( $n=16$ études), les platines $(n=7)$ et l'alpha-fluoro-béta-alanine, un métabolite urinaire du 5 -fluorouracile $(n=3)$. La proportion de travailleurs qui ont étés déclarés positifs s'étendait de $0 \%$ ( $n=10$ études) à $100 \%(n=4)$. Si l'on ne retient que les études permettant de calculer le taux de travailleurs comportant au moins un prélèvement urinaire positif $(n=23)$, la proportion totale était de $21 \%(173 / 809$ travailleurs, toutes méthodes et tous médicaments confondus).

Conclusion : Vingt-quatre études de surveillance urinaire ont été réalisées au sein de sept pays entre 2010 et 2015. Dans plusieurs études, aucune trace de médicaments n'a été mesurée dans l'urine.

Mots-clés : surveillance biologique, urine, médicaments dangereux, exposition professionnelle

\begin{abstract}
Background: There is increasing evidence that traces of hazardous drugs occur in the urine of health care professionals who are exposed to these drugs.

Objective: To review the scientific literature regarding urinary monitoring of health care professionals exposed to antineoplastic drugs through their work.

Data Sources: A search of PubMed using the Medical Subject Headings "occupational exposure" and "antineoplastic agents" and of Google Scholar using the terms "antineoplastic", "urine", and "occupational exposure".
\end{abstract}

Study Selection and Data Extraction: The analysis covered all articles in English or French pertaining to health care professionals exposed to hazardous drugs in the workplace, published from January 1, 2010, to December 31, 2015. Articles that did not discuss the results of urine tests and those concerning veterinarians, as well as literature reviews, editorials, letters to the editor, and conference abstracts, were excluded.

Data Synthesis: Twenty-four articles were retained. The studies were conducted in 52 health care institutions in 7 countries. They included 826 workers exposed to hazardous drugs and 175 controls, specifically nurses ( $n=16$ studies), pharmacists $(n=10)$, pharmacy technicians $(n=8)$, physicians $(n=7)$, health care aides $(n=2)$, and others $(n=8)$. Various analytical methods were used to quantify the presence of 13 hazardous drugs, primarily cyclophosphamide ( $n=16$ studies), platinumbased drugs $(n=7)$, and alpha-fluoro-beta-alanine, a urine metabolite derived from 5-fluorouracil $(n=3)$. The proportion of workers with positive results ranged from $0 \%(n=10$ studies) to $100 \%(n=4)$. Considering only those studies that allowed calculation of the rate of workers with at least one positive urine sample $(n=23)$, the total proportion was $21 \%$ (173/809 workers, for all methods and drugs combined).

Conclusion: Twenty-four studies on urine monitoring were conducted in 7 countries between 2010 and 2015. In several studies, no traces of drugs were detected in urine.

Keywords: biomonitoring, urine, hazardous drugs, occupational exposure

\section{J. Can. Pharm. Hosp. 2016;69(5):376-87}




\section{CONTEXTE}

$\mathrm{B}_{\mathrm{d}}^{\mathrm{i}}$

ien que le terme " médicament dangereux " n'ait pas encore d'assise juridique au Canada, le National Institute for Occupational Safety and Health (NIOSH) le définit comme étant un médicament qui présente un potentiel cancérogène, mutagène, tératogène, toxique pour la reproduction, toxique pour les organes, génotoxique ou similaire à un autre médicament dangereux ${ }^{1}$. Cet organisme met périodiquement à jour une liste des médicaments dangereux. En 2014, elle contient une majorité de médicaments antinéoplasiques $(97 / 184,53 \%)^{2}$.

Il existe trois types de surveillance de l'exposition professionnelle aux médicaments dangereux : la surveillance environnementale, la surveillance médicale et la surveillance biologique. La surveillance environnementale est effectuée dans le cadre de travaux de recherche ou de programmes structurés de surveillance depuis au moins deux décennies. Elle permet la détection de traces de médicaments dangereux sur différentes surfaces. Au Québec, l'Association paritaire pour la santé et la sécurité du travail du secteur affaires sociales au Québec (ASSTSAS) recommande de pratiquer une détection annuellement ainsi qu'à chaque changement important de pratique ou d'aménagement ${ }^{3}$. L'Ordre des pharmaciens du Québec recommande également une surveillance environnementale de la contamination chimique deux fois par année ${ }^{4}$. La surveillance médicale permet la détection de signes et de symptômes potentiellement associés à une exposition professionnelle. Comme cette mesure de surveillance n'est pas spécifique, l'ASSTSAS ne recommande pas de l'effectuer. Le NIOSH recommande néanmoins de procéder à la surveillance médicale. ${ }^{5}$.

Enfin, la surveillance biologique permet la détection et la quantification de traces de médicaments dangereux dans les urines ou le sang. Si l'élimination complète des traces de médicaments dangereux sur les surfaces est difficile à assurer en tout temps, on devrait éviter leur présence dans les liquides biologiques et instaurer rapidement des mesures correctrices en cas de résultats positifs. En effet, certaines études ont décelé un effet néfaste sur la fonction de reproduction des travailleurs exposés à ces produits dans le cadre de leur travail ${ }^{6}$ et des effets sur les chromosomes ${ }^{7}$.

Depuis une décennie, plusieurs études ont été menées au Canada afin de quantifier les traces de médicaments dangereux sur les surfaces ${ }^{8-17}$.

Notre équipe de recherche s'intéresse à la protection des travailleurs potentiellement exposés aux médicaments dangereux. Dans ce cadre, nous avons mené une revue de la littérature scientifique sur la surveillance urinaire afin d'établir un portrait des méthodes actuellement utilisées et du degré de contamination mentionné dans la littérature scientifique.

\section{OBJECTIF}

L'objectif principal vise à présenter une revue de la littérature scientifique sur la surveillance urinaire de professionnels de la santé exposés aux antinéoplasiques dans le cadre de leur travail.

\section{SOURCES DE DONNÉES}

Afin de recenser tous les articles pertinents portant sur la surveillance urinaire de professionnels de la santé exposés aux antinéoplasiques dans le cadre de leur travail, nous avons consulté PubMed en utilisant la stratégie suivante : (" antineoplastic agents" [Pharmacological Action] OR " antineoplastic agents " $[\mathrm{MeSH}$ Terms] OR (" antineoplastic " [All Fields] AND " agents" [All Fields]) OR " antineoplastic agents " [All Fields]) AND ("occupational exposure» [MeSH Terms $]$ OR («occupational» $[$ All Fields] AND " exposure" [All Fields]) OR « occupational exposure» [All Fields]). De plus, nous avons consulté Google Scholar en utilisant la stratégie suivante : "antineoplastic ", " urine " et "occupational exposure". Enfin, nous avons mené une recherche manuelle à partir des listes de références des articles retenus.

\section{SÉLECTION DES ÉTUDES ET EXTRACTION DES DONNÉES}

La revue de littérature ciblait tous les articles en anglais et en français ayant trait aux professionnels de santé potentiellement exposés à des médicaments dangereux dans le cadre de leur travail, publiés entre le $1^{\text {er }}$ janvier 2010 et le 31 décembre 2015. Une première sélection des articles pertinents a été effectuée à partir des titres et des résumés. Ensuite, la consultation des textes complets des articles a permis leur inclusion ou non dans notre corpus. Nous avons exclu les articles ne comportant pas de résultats urinaires, les études portant sur les vétérinaires ainsi que les revues de littérature, les éditoriaux, les lettres à la rédaction et les résumés de congrès.

Placé sous la supervision du chercheur, un premier assistant de recherche a procédé à la sélection des études et à l'extraction des données. Un second assistant de recherche a répété l'ensemble de la démarche. Les désaccords ont été résolus par consensus.

L'extraction des données pertinentes portait sur les éléments suivants : auteurs, pays, année, type de l'étude (monocentrique ou multicentrique), taille de la population (exposé et témoin), répartition des participants selon leur profession, méthodes analytiques, médicaments ciblés, limites de détection et de quantification, détails sur les prélèvements urinaires, présence ou non d'une description des pratiques de manipulation (équipement de protection) et d'une description de l'exposition (type d'exposition, quantité d'antinéoplasiques manipulée), type et période de collecte urinaire, nombre de prélèvements, nombre et proportion de prélèvements positifs, résultats des concentrations urinaires, nombre et proportion de travailleurs qui ont étés déclarés positifs. Seules les données descriptives ont été recueillies.

\section{SYNTHÈSE DES DONNÉES}

La recherche sur PubMed a mis au jour 265 articles. Trente-neuf articles ont été sélectionnés selon leur titre. Puis 18 articles ont été sélectionnés selon le résumé. Après 
consultation des textes complets, quatre articles ont été exclus pour les motifs suivants : dans une autre langue que l'anglais ou le français (2), hors du sujet (1) et revue de littérature (1). Une recherche manuelle a permis d'ajouter dix articles aux 14 retenus précédemment. Finalement, la collecte des données a porté sur 24 articles (tableau 1$)^{18-41}$.

Les études retenues présentaient les travaux de 21 auteurs provenant de sept pays soit: Japon $(n=7)$, Italie $(n=6)$, France $(n=4)$, Canada $(n=3)$, États-Unis $(n=2)$, Belgique $(n=1)$ et Suède $(n=1)$. Elles ont été menées au sein de 52 établissements de santé. Neuf étaient multicentriques, et trois présentaient des mesures répétées dans le temps. Elles regroupaient 826 travailleurs exposés à des médicaments dangereux et 175 témoins (sans compter les données incomplètes d'une étude). Les acteurs de la santé étudiés se répartissaient dans les catégories professionnelles suivantes : infirmiers ( $n=16$ études), pharmaciens $(n=10)$, assistants techniques en pharmacie $(n=8)$, médecins $(n=7)$, aides-soignants $(n=2)$ et autres catégories $(n=8)$. Neuf méthodes analytiques différentes ont été utilisées : chromatographie en phase gazeuse couplée à la spectrométrie de masse (GC-MS) ( $n=7$ études), chromatographie en phase liquide à haute performance couplée à la spectrométrie de masse en tandem (HPLC-MS/MS) $(n=5)$, spectrométrie de masse à plasma à couplage inductif (ICP-MS) $(n=5)$, chromatographie en phase gazeuse couplée à la spectrométrie de masse en tandem (GC-MS/MS) ( $n=4)$, chromatographie en phase liquide couplée à la spectrométrie de masse en tandem (LC-MS/MS) $(n=3)$, chromatographie en phase liquide à haute performance couplée à la détection en fluorescence (HPLC-FL) $(n=1)$, chromatographie en phase liquide à haute performance interfacée à un détecteur ultraviolet (HPLC-UV) $(n=1)$, spectrométrie de masse à plasma à couplage inductif à secteur magnétique (ICP-SFMS) $(n=1)$, voltamétrie $(n=1)$. Tous médicaments confondus, les limites de détection s'étendaient de $1,5 \times 10^{-3}$ à $1,2 \mathrm{ng} / \mathrm{mL}$, et les limites de quantification variaient de $2 \times 10^{-3}$ à $2,0 \mathrm{ng} / \mathrm{mL}$.

Douze médicaments dangereux différents ont été quantifiés, ainsi qu'un métabolite, soit : cyclophosphamide $(n=16)$, platines $(n=7)$, alpha-fluoro-béta-alanine, métabolite urinaire du 5-FU $(n=3)$, doxorubicine $(n=2)$, épirubicine $(n=2)$, ifosfamide $(n=2)$, 5-fluoro-uracile (5-FU) $(n=1)$, gemcitabine $(n=1)$, méthotrexate $(n=1)$, étoposide $(n=1)$, docétaxel $(n=1)$, pémétrexed $(n=1)$, paclitaxel $(n=1)$. Quinze études ont quantifié la présence d'un seul médicament, et neuf ont quantifié

Tableau 1 (partie 1 de 7). Profil des résultats de 24 études de surveillance urinaire ${ }^{18-41}$ menées sur des professionnels de la santé exposés aux antinéoplasiques et publiées entre le 1er janvier 2010 et le 31 décembre 2015*

\begin{tabular}{|c|c|c|c|c|c|c|}
\hline Étude et Pays & $\begin{array}{c}\text { Type } \\
\text { (n centres), } \\
\text { Population } \\
\text { (n travailleurs), } \\
\text { Professions } \\
\text { (n travailleurs) }\end{array}$ & $\begin{array}{l}\text { Méthode } \\
\text { lédicaments } \\
\text { - Limites } \\
\text { (ng/mL) }\end{array}$ & $\begin{array}{l}\text { Prélèvements urinaires } \\
\text { Pratiques de manipulation et exposition } \\
\text { - Type de collecte } \\
\text { - Période de collecte } \\
\text { - Prélèvements }(n)\end{array}$ & $\begin{array}{l}\text { Prélèvements } \\
\text { positifs } \\
n(\%)\end{array}$ & $\begin{array}{c}\text { Travailleurs } \\
\text { positifs } \\
n(\%)\end{array}$ & $\begin{array}{l}\text { Concentrations } \\
(\mathrm{ng} / \mathrm{mL})\end{array}$ \\
\hline $\begin{array}{l}\text { Friese et al. } \\
(2015)^{18} \\
\text { États-Unis }\end{array}$ & $\begin{array}{l}\text { Monocentrique (1) } \\
\text { Population } \\
\text { - Exposés (17) } \\
\text { (dont } 9 \\
\text { personnes } \\
\text { exposées à un } \\
\text { déversement) } \\
\text { Professions } \\
\text { - Infirmiers (17) }\end{array}$ & $\begin{array}{l}\text { LC-MS/MS } \\
\text { Étoposide } \\
\text { - LIQ : 0,02 } \\
\text { - LD : ND } \\
\text { Docetaxel } \\
\text { - LIQ : 0,025 } \\
\text { - LD : ND } \\
\text { Pemetrexed } \\
\text { - LIQ : 0,10 } \\
\text { - LD : ND }\end{array}$ & $\begin{array}{l}\text { - Pratiques de manipulation et exposition } \\
\text { décrites grâce à un questionnaire prospectif } \\
\text { pendant } 6 \text { mois puis un questionnaire } \\
\text { rétrospectif } \\
\text { - Type : collecte urinaire de } 8 \mathrm{~h} \\
\text { - Urine à partir de } 4 \mathrm{~h} \text { après exposition } \\
\text { au déversement } \\
\text { - Urine des } 4 \text { dernières heures du quart } \\
\text { de travail et urine des } 4 \text { h suivantes } \\
\text { sans déversement } \\
\text { - Période : } 6 \text { mois } \\
\text { - } 17 \text { prélèvements }\end{array}$ & $\begin{array}{l}\text { Exposés : } \\
\text { - Étoposide : } \\
\text { 1/8 (13\%) } \\
\text { - Docetaxel : } \\
\text { 7/9 (78 \%) } \\
\text { - Pemetrexed } \\
\text { 0/9 (0 \%) }\end{array}$ & $\begin{array}{l}\text { Exposés : } \\
8 / 17(47 \%)\end{array}$ & $\begin{array}{l}\text { Exposés : } \\
\text { - Étoposide : } \\
\text { Min. : LD } \\
\text { Max. : LIQ } \\
\text { - Docetaxel : } \\
\text { Min. : LD } \\
\text { Max. : 0,58 } \\
\text { - Pemetrexed } \\
\text { < LD }\end{array}$ \\
\hline $\begin{array}{l}\text { Hon et al. } \\
(2015)^{19} \\
\text { Canada }\end{array}$ & $\begin{array}{l}\text { Multicentrique (6) } \\
\text { Population } \\
\text { - Exposés (103 au } \\
\text { prélevement \#1 } \\
\text { dont } 98 \text { ont } \\
\text { participé au } \\
\text { prélèvement \#2) } \\
\text { Professions (phase I } \\
\text { c. phase II) } \\
\text { - ATP (21 c. 21) } \\
\text { - Infirmiers (29 c. 28) } \\
\text { - Pharmaciens } \\
\text { (16 c. } 16) \\
\text { - Autres (37 c. 33) }\end{array}$ & $\begin{array}{l}\text { HPLC-MS/MS } \\
\text { CP } \\
\text { - LD : 0,05 } \\
\text { - LQ : ND } \\
\text { 8) }\end{array}$ & $\begin{array}{l}\text { - Pratiques de manipulation non décrites. } \\
\text { Exposition décrite grâce à un questionnaire } \\
\text { puis lors d'un entretien avec un membre } \\
\text { de l'équipe après la collecte urinaire. } \\
\text { - Type : collecte urinaire de } 24 \text { h } \\
\text { - Période : } 2 \text { collectes à } 3 \text { semaines } \\
\text { d'intervalle entre juin } 2010 \text { et } \\
\text { février } 2011 \\
\text { - } 201 \text { prélèvements }\end{array}$ & $\begin{array}{l}\text { Exposés: } \\
111 / 201 \\
(55 \%)\end{array}$ & $\begin{array}{l}\text { Exposés : } \\
\text { Prélevement } \\
\# 1: 41 / 103 \\
(40 \%) \\
\text { Prélèvement } \\
\# 2: 70 / 98 \\
(71 \%)\end{array}$ & $\begin{array}{l}\text { Exposés : } \\
\text { Max. : 2,37 } \\
\text { (chez un ATP) } \\
\text { Moyenne : } \\
0,156 \\
75^{e} \text { percentile : } \\
0,129\end{array}$ \\
\hline
\end{tabular}


Tableau 1 (partie 2 de 7). Profil des résultats de 24 études de surveillance urinaire ${ }^{1841}$ menées sur des professionnels de la santé exposés aux antinéoplasiques et publiées entre le 1" janvier 2010 et le 31 décembre 2015*

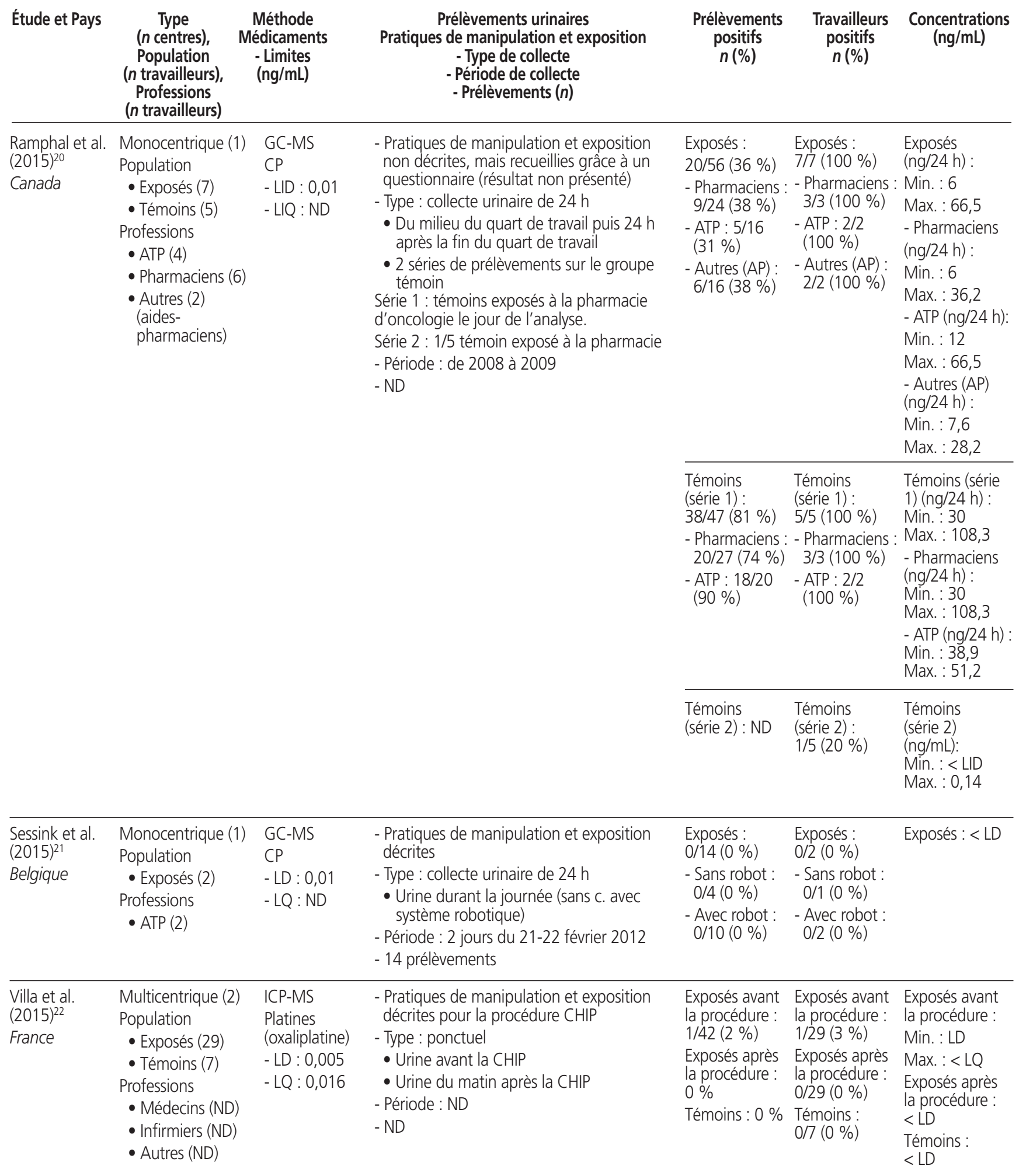


Tableau 1 (partie 3 de 7). Profil des résultats de 24 études de surveillance urinaire ${ }^{1841}$ menées sur des professionnels

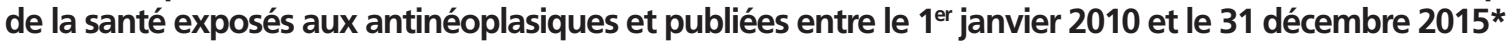

\begin{tabular}{|c|c|c|c|c|c|c|}
\hline Étude et Pays & $\begin{array}{c}\text { Type } \\
\text { (n centres), } \\
\text { Population } \\
\text { (n travailleurs), } \\
\text { Professions } \\
\text { (n travailleurs) }\end{array}$ & $\begin{array}{l}\text { Méthode } \\
\text { Médicaments } \\
\text { - Limites } \\
\text { (ng/mL) }\end{array}$ & $\begin{array}{l}\text { Prélèvements urinaires } \\
\text { Pratiques de manipulation et exposition } \\
\text { - Type de collecte } \\
\text { - Période de collecte } \\
\text { - Prélèvements }(n)\end{array}$ & $\begin{array}{c}\text { Prélèvements } \\
\text { positifs } \\
n(\%)\end{array}$ & $\begin{array}{c}\text { Travailleurs } \\
\text { positifs } \\
n(\%)\end{array}$ & $\begin{array}{l}\text { Concentrations } \\
(\mathrm{ng} / \mathrm{mL})\end{array}$ \\
\hline $\begin{array}{l}\text { Caneparo } \\
\text { et al. }(2014)^{23} \\
\text { Italie }\end{array}$ & $\begin{array}{l}\text { Monocentrique (1) } \\
\text { Population } \\
\text { - Exposés (5) } \\
\text { Professions } \\
\text { - Infirmiers (1) } \\
\text { - Médecins (4) }\end{array}$ & $\begin{array}{l}\text { ICP-MS } \\
\text { Platines } \\
\text { (cisplatine) } \\
\text { - LD : } 1 \\
\text { - LQ : ND }\end{array}$ & $\begin{array}{l}\text { - Pratiques de manipulation et exposition } \\
\text { décrites pour la procédure CHIP } \\
\text { - Type : ponctuel et répété } \\
\text { - } 1 \text { échantillon avant la CHIP } \\
\text { - } 1 \text { échantillon après la CHIP } \\
\text { - } 1 \text { échantillon } 24 \text { h après la CHIP } \\
\text { - Période : deux jours non consécutifs } \\
\text { - } 30 \text { prélèvements }\end{array}$ & $\begin{array}{l}\text { Exposés : } \\
0 / 30(0 \%)\end{array}$ & $\begin{array}{l}\text { Exposés : } \\
0 / 5(0 \%)\end{array}$ & Exposés : <LD \\
\hline $\begin{array}{l}\text { Ramphal et al. } \\
(2014)^{24} \\
\text { Canada }\end{array}$ & $\begin{array}{l}\text { Monocentrique (1) } \\
\text { Population } \\
\text { - Exposés (41) } \\
\text { - Témoins (49) } \\
\text { Professions } \\
\text { - Infirmiers (80) } \\
\text { - Autres (10) }\end{array}$ & $\begin{array}{l}\text { GC-MS } \\
\text { CP } \\
- \text { LID: } 0,01 \\
- \text { LIQ : } 0,01\end{array}$ & $\begin{array}{l}\text { - Pratiques de manipulation et exposition } \\
\text { de la journée de recueil décrites et recueillies } \\
\text { grâce à un questionnaire } \\
\text { - Type : collecte urinaire de } 28-30 \mathrm{~h} \\
\text { - Du milieu du quart de travail puis } 24 \mathrm{~h} \\
\text { après la fin du quart de travail (exposés } \\
\text { et témoins infirmiers) } \\
\text { - Type : collecte urinaire de } 24 \mathrm{~h} \\
\text { - Selon convenance (témoins } \\
\text { communautaires) } \\
\text { - Période : } 4 \text { mois } \\
\text { - } 90 \text { prélèvements }\end{array}$ & $\begin{array}{l}\text { Exposés : } \\
\text { 14/41 (34 \%) } \\
\text { Témoins } \\
\text { infirmiers : } \\
13 / 39(33 \%) \\
\text { Témoins } \\
\text { com. : } 0 / 10 \\
(0 \%)\end{array}$ & $\begin{array}{l}\text { Exposés : } \\
\text { 14/41 (34 \%) } \\
\text { Témoins } \\
\text { infirmiers : } \\
13 / 39(33 \%) \\
\text { Témoins } \\
\text { com. : } \\
0 / 10(0 \%)\end{array}$ & $\begin{array}{l}\text { Exposés } \\
(\mathrm{ng} / 24 \mathrm{~h}) \\
(n=13) \\
\text { Min. : } 12 \\
\text { Max. : } 474 \\
\text { Moyenne : } 162 \\
\text { Médiane : } 39 \\
\text { Témoins } \\
\text { infirmiers } \\
\text { (ng/24 h) : } \\
\text { Min. : } 18 \\
\text { Max. : } 286 \\
\text { Moyenne : } 88 \\
\text { Médiane : } 52 \\
\text { Témoins } \\
\text { com. : < LID }\end{array}$ \\
\hline \multirow[t]{2}{*}{$\begin{array}{l}\text { Miyake et al. } \\
(2013)^{25} \\
\text { Japon }\end{array}$} & $\begin{array}{l}\text { Monocentrique (1) } \\
\text { Population } 2007 \\
\text { - Exposés (4) } \\
\text { Professions } \\
\text { - Pharmaciens (4) }\end{array}$ & $\begin{array}{l}\text { GC-MS/MS } \\
\text { CP } \\
-L D: 0,01 \\
-L Q: N D\end{array}$ & $\begin{array}{l}\text { - Pratiques de manipulation et exposition } \\
\text { décrites } \\
\text { - Type : collecte urinaire de } 24 \mathrm{~h} \\
\text { - Urine du début de la journée de } \\
\text { manipulation au lendemain matin } \\
\text { - Période : } 30 \text { août au } 11 \text { septembre } 2007 \\
\text { - } 34 \text { prélèvements }\end{array}$ & $\begin{array}{l}\text { Exposés avant } \\
\text { système clos : } \\
26 / 34 \text { (77 \%) }\end{array}$ & $\begin{array}{l}\text { Exposés avant } \\
\text { système } \\
\text { fermé : } \\
4 / 4(100 \%)\end{array}$ & $\begin{array}{l}\text { Exposés avant } \\
\text { système clos } \\
\text { (ng/24 h): } \\
4 \text { collectes } \\
\text { positives, soit } \\
34,9 ; 27 ; 56,5 ; \\
71,3 \\
\text { Moyenne : } 47,4\end{array}$ \\
\hline & $\begin{array}{l}\text { Monocentrique (1) } \\
\text { Population } \\
\text { 2008-2009 } \\
\text { - Exposés (4) } \\
\text { Professions } \\
\text { - Pharmaciens (4) }\end{array}$ & & $\begin{array}{l}\text { - Pratiques de manipulation et exposition } \\
\text { décrites } \\
\text { - Type : collecte urinaire de } 24 \mathrm{~h} \\
\text { - Urine du début de la journée de } \\
\text { manipulation au lendemain matin } \\
\text { - Période : } 7 \text { novembre } 2008 \text { au } \\
17 \text { mars } 2009 \\
\text { - } 31 \text { prélèvements }\end{array}$ & $\begin{array}{l}\text { Exposés après } \\
\text { système clos : } \\
2 / 31(6 \%)\end{array}$ & $\begin{array}{l}\text { Exposés après } \\
\text { système fermé : } \\
2 / 4(50 \%)\end{array}$ & $\begin{array}{l}\text { Exposés après } \\
\text { : système clos } \\
\text { (ng/24 h) : } \\
2 \text { collectes } \\
\text { positives, soit } \\
6,4 ; 7,8 \\
\text { Moyenne : 3,6 }\end{array}$ \\
\hline $\begin{array}{l}\text { Kopp et al. } \\
(2013)^{26} \\
\text { France }\end{array}$ & $\begin{array}{l}\text { Multicentrique (2) } \\
\text { Population } \\
\text { - Exposés (10) } \\
\text { - Témoins (5) } \\
\text { Professions } \\
\text { - Autre (personnel } \\
\text { pharmaceutique) } \\
\text { (15) }\end{array}$ & $\begin{array}{l}\text { Voltamétrie } \\
\text { Platines } \\
\text { - LD : ND } \\
\text { - LQ : 0,002 } \\
\text { el } \\
\text { e) }\end{array}$ & $\begin{array}{l}\text { - Pratiques de manipulation et exposition } \\
\text { décrites } \\
\text { - Type : ponctuel et répété } \\
\text { - } 1 \text { échantillon le lundi avant le début du } \\
\text { quart de travail } \\
\text { - } 1 \text { échantillon le vendredi avant le début } \\
\text { du quart de travail } \\
\text { - } 1 \text { échantillon le vendredi après le quart } \\
\text { de travail } \\
\text { - Période : } 2 \text { jours non consécutifs } \\
\text { - } 37 \text { prélèvements (30 exposés c. } 7 \text { témoins) }\end{array}$ & $\begin{array}{l}\text { Toutes } \\
\text { populations } \\
\text { confondues : } \\
37 / 37(100 \%)\end{array}$ & $\begin{array}{l}\text { Exposés : } \\
\text { 10/10 (100 \%) } \\
\text { Témoins : } \\
\text { 5/5 (100 \%) }\end{array}$ & $\begin{array}{l}\text { Toutes } \\
\text { populations } \\
\text { confondues : } \\
\text { Min : } 0,8 \times 10^{-3} \\
\text { Max : } 0,0046\end{array}$ \\
\hline
\end{tabular}


Tableau 1 (partie 4 de 7). Profil des résultats de 24 études de surveillance urinaire ${ }^{1841}$ menées sur des professionnels

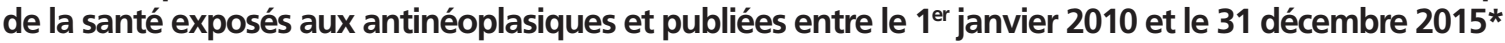

\begin{tabular}{|c|c|c|c|c|c|c|}
\hline Étude et Pays & $\begin{array}{c}\text { Type } \\
\text { (n centres), } \\
\text { Population } \\
\text { (n travailleurs), } \\
\text { Professions } \\
\text { (n travailleurs) }\end{array}$ & $\begin{array}{l}\text { Méthode } \\
\text { Médicaments } \\
\text { - Limites } \\
\text { (ng/mL) }\end{array}$ & $\begin{array}{l}\text { Prélèvements urinaires } \\
\text { Pratiques de manipulation et exposition } \\
\text { - Type de collecte } \\
\text { - Période de collecte } \\
\text { - Prélèvements }(n)\end{array}$ & $\begin{array}{c}\text { Prélèvements } \\
\text { positifs } \\
n(\%)\end{array}$ & $\begin{array}{l}\text { Travailleurs } \\
\text { positifs } \\
n(\%)\end{array}$ & $\begin{array}{l}\text { Concentrations } \\
(\mathrm{ng} / \mathrm{mL})\end{array}$ \\
\hline \multirow[t]{2}{*}{$\begin{array}{l}\text { Yoshida } \\
\text { et al. }(2013)^{28} \\
\text { Japon }\end{array}$} & $\begin{array}{l}\text { Monocentrique (1) } \\
\text { Population } \\
2007-2008 \\
\text { - Exposés (8) } \\
\text { Professions } \\
\text { - Pharmaciens (8) }\end{array}$ & \multirow[t]{2}{*}{$\begin{array}{l}\text { GC-MS } \\
\text { CP } \\
- \text { LD : } 0,003 \\
- \text { LQ : } 0,02 \\
\text { FBAL } \\
- \text { LD : } 0,016 \\
- \text { LQ : } 0,04\end{array}$} & $\begin{array}{l}\text { - Pratiques de manipulation décrites sous } \\
\text { forme de score et exposition décrite pour } \\
\text { chaque période de collecte } \\
\text { - Type : collecte urinaire de } 24 \mathrm{~h} \\
\text { - Avant la préparation jusqu'au lendemain } \\
\text { - Période : octobre } 2007 \text {, février } 2008 \text { et } \\
\text { décembre } 2008 \text { (score de qualité < } 80 \% \text { ) } \\
\text { - } 11 \text { prélèvements }\end{array}$ & $\begin{array}{l}\text { 2007-2008: } \\
\text { CP: } \\
\text { 5/11 (45 \%) } \\
\text { - FBAL: } \\
6 / 11(55 \%)\end{array}$ & $\begin{array}{l}\text { 2007-2008: } \\
\text { ND }\end{array}$ & $\begin{array}{l}\text { 2007-2008: } \\
\text { - CP : Max. } \\
\sim 40 \mathrm{ng} / 24 \mathrm{~h} \\
\text { - FBAL : Max. } \\
\sim 280 \mathrm{ng} / 24 \mathrm{~h}\end{array}$ \\
\hline & $\begin{array}{l}\text { Monocentrique (1) } \\
\text { Population } 2011 \\
\text { - Exposés (4) } \\
\text { Professions } \\
\text { - Pharmaciens (4) }\end{array}$ & & $\begin{array}{l}\text { - Pratiques de manipulation décrites sous } \\
\text { forme de score et exposition décrite pour } \\
\text { chaque période de collecte } \\
\text { - Type : collecte urinaire de } 24 \mathrm{~h} \\
\text { - Avant la préparation jusqu'au lendemain } \\
\text { - Période : février } 2011 \text { et juin } 2011 \\
\text { (score de qualité > } 80 \% \text { ) } \\
\text { - } 6 \text { prélèvements }\end{array}$ & $\begin{array}{l}2011: \\
\text { - CP : } 0 / 6(0 \%) \\
\text { - FBAL : } \\
1 / 6(17 \%)\end{array}$ & $\begin{array}{l}2011: \\
\text { - CP : 0/4 (0 \%) } \\
\text { - FBAL: } \\
1 / 4(25 \%)\end{array}$ & $\begin{array}{l}2011: \\
\text { - CP : < LD } \\
\text { - FBAL: Max. } \\
\sim 40 \text { ng/24 h }\end{array}$ \\
\hline \multirow[t]{3}{*}{$\begin{array}{l}\text { Sabatini } \\
\text { et al. }(2012)^{27} \\
\text { Italie }\end{array}$} & $\begin{array}{l}\text { Monocentrique (1) } \\
\text { Population } 2001 \\
\text { - Exposés (50) } \\
\text { - Témoins (50) } \\
\text { Professions } 2001 \\
\text { - Infirmiers (100) }\end{array}$ & \multirow[t]{3}{*}{$\begin{array}{l}\text { LC-MS/MS } \\
\text { CP } \\
\text { - LD : 0,04 } \\
\text { - LQ : ND } \\
\text { Méthotrexate : } \\
\text { - LD : 0,2 } \\
\text { - LQ : ND }\end{array}$} & $\begin{array}{l}\text { - Pratiques de manipulation et exposition } \\
\text { non décrites } \\
\text { - Type : ponctuel } \\
\text { - } 1 \text { échantillon à la fin du quart de travail } \\
\quad \text { au moins } 4 \mathrm{~h} \text { après la manipulation } \\
\text { - Période : } 2001 \\
\text { - } 100 \text { prélèvements }\end{array}$ & $\begin{array}{l}\text { Exposés : } \\
\text { CP : } \\
6 / 50(12 \%) \\
\text { Méthotrexate : } \\
8 / 50(16 \%)\end{array}$ & $\begin{array}{l}\text { Exposés : } \\
18 / 50 \text { (36 \%) }\end{array}$ & $\begin{array}{l}\text { Exposés : } \\
\text { - CP : } \\
\text { Min. : } 0,06 \\
\text { Max. : } 10,00 \\
\text { - Méthotrexate } \\
\text { Min. : } 0,30 \\
\text { Max. : 2,01 }\end{array}$ \\
\hline & $\begin{array}{l}\text { Monocentrique (1) } \\
\text { Population } 2005 \\
\text { - Exposés (81) } \\
\text { Professions } 2005 \\
\text { - ATP (ND) } \\
\text { - Infirmiers (ND) }\end{array}$ & & $\begin{array}{l}\text { - Pratiques de manipulation et exposition } \\
\text { non décrites } \\
\text { - Type : ponctuel } \\
\text { - } 1 \text { échantillon à la fin du quart de travail } \\
\text { au moins } 4 \mathrm{~h} \text { après la manipulation } \\
\text { - Période : } 2005 \\
\text { - } 81 \text { prélèvements }\end{array}$ & $\begin{array}{l}\text { Exposés : } \\
\text { CP : } 7 / 81 \text { (9 \%) } \\
\text { Méthotrexate : } \\
3 / 81(4 \%)\end{array}$ & $\begin{array}{l}\text { Exposés : } \\
\text { 9/81 (11\%) }\end{array}$ & $\begin{array}{l}\text { Exposés: } \\
- \text { CP : } \\
\text { Min. : 0,08 } \\
\text { Max. : 0,25 } \\
\text { - Méthotrexate : } \\
\text { Min. : 0,40 } \\
\text { Max. : 0,58 }\end{array}$ \\
\hline & $\begin{array}{l}\text { Monocentrique (1) } \\
\text { Population } 2010 \\
\text { - Exposés (54) } \\
\text { Professions } 2010 \\
\text { - ATP (4) } \\
\text { - Infirmiers (50) }\end{array}$ & & $\begin{array}{l}\text { - Pratiques de manipulation et exposition } \\
\text { non décrites } \\
\text { - Type : ponctuel } \\
\text { - } 1 \text { échantillon à la fin du quart de travail } \\
\text { au moins } 4 \text { h après la manipulation } \\
\text { - Période : } 2010 \\
\text { - } 54 \text { prélèvements }\end{array}$ & $\begin{array}{l}\text { Exposés : } \\
0 / 54(0 \%)\end{array}$ & $\begin{array}{l}\text { Exposés : } \\
0 / 54(0 \%)\end{array}$ & $\begin{array}{l}\text { Exposés: } \\
<\mathrm{LD}\end{array}$ \\
\hline $\begin{array}{l}\text { Sottani } \\
\text { et al. }(2012)^{29} \\
\text { Italie }\end{array}$ & $\begin{array}{l}\text { Multicentrique (4) } \\
\text { Population } \\
\text { - Exposés (36) } \\
\text { Professions } \\
\text { - ATP (11) } \\
\text { - Infirmiers (25) }\end{array}$ & $\begin{array}{l}\text { HPLC-MS/MS } \\
\text { CP, ifosfamide } \\
\text { et gemcitabine } \\
\text { - LID : ND } \\
\text { - LIQ : } 0,2\end{array}$ & $\begin{array}{l}\text { - Pratiques de manipulation et exposition } \\
\text { de la journée de recueil décrites et } \\
\text { recueillies grâce à un questionnaire } \\
\text { - Type : ponctuel } \\
\text { - Urines avant quart de travail } \\
\text { - Urines après quart de travail d'au moins } 6 \mathrm{~h} \\
\text { - Période : } 2009 \\
\text { - ND }\end{array}$ & Exposés : ND & $\begin{array}{l}\text { Exposés : } \\
0 / 36(0 \%)\end{array}$ & Exposés : < LID \\
\hline
\end{tabular}


Tableau 1 (partie 5 de 7). Profil des résultats de 24 études de surveillance urinaire ${ }^{1841}$ menées sur des professionnels de la santé exposés aux antinéoplasiques et publiées entre le 1" janvier 2010 et le 31 décembre 2015*

\begin{tabular}{|c|c|c|c|c|c|c|}
\hline Étude et Pays & $\begin{array}{c}\text { Type } \\
\text { (n centres), } \\
\text { Population } \\
\text { (n travailleurs), } \\
\text { Professions } \\
\text { ( } n \text { travailleurs) } \\
\end{array}$ & $\begin{array}{l}\text { Méthode } \\
\text { Médicaments } \\
\text { - Limites } \\
\text { (ng/mL) }\end{array}$ & $\begin{array}{l}\text { Prélèvements urinaires } \\
\text { Pratiques de manipulation et exposition } \\
\text { - Type de collecte } \\
\text { - Période de collecte } \\
\text { - Prélèvements }(n)\end{array}$ & $\begin{array}{l}\text { Prélèvements } \\
\text { positifs } \\
n(\%)\end{array}$ & $\begin{array}{l}\text { Travailleurs } \\
\text { positifs } \\
n(\%)\end{array}$ & $\begin{array}{l}\text { Concentrations } \\
\text { (ng/mL) }\end{array}$ \\
\hline $\begin{array}{l}\text { Hama et al. } \\
(2012)^{30} \\
\text { Japon }\end{array}$ & $\begin{array}{l}\text { Monocentrique (1) } \\
\text { Population } \\
\text { - Exposé (1) } \\
\text { Professions } \\
\text { - Pharmacien (1) }\end{array}$ & $\begin{array}{l}\text { GC-MS/MS } \\
\text { CP } \\
- \text { LD : 0,01 } \\
\text { - LQ : ND }\end{array}$ & $\begin{array}{l}\text { - Pratiques de manipulation et exposition } \\
\text { décrites pour la période de collecte } \\
\text { - Type : collecte urinaire de } 29 \text { heures } \\
\text { - Période : } 2 \text { jours consécutifs } \\
\text { - } 7 \text { prélèvements }\end{array}$ & $\begin{array}{l}\text { Exposé : } \\
1 / 7(14 \%)\end{array}$ & $\begin{array}{l}\text { Exposé : } \\
1 / 1(100 \%)\end{array}$ & $\begin{array}{l}\text { Exposé } \\
\text { (ng/24 h) : 13,5 }\end{array}$ \\
\hline $\begin{array}{l}\text { Sugiura et al. } \\
(2011)^{31} \\
\text { Japon }\end{array}$ & $\begin{array}{l}\text { Monocentrique (1) } \\
\text { Population } \\
\text { - Exposés (10) } \\
\text { Professions } \\
\text { - Pharmaciens (3) } \\
\text { - Infirmiers (4) } \\
\text { - Médecins (3) }\end{array}$ & $\begin{array}{l}\text { GC-MS/MS } \\
\text { CP } \\
- \text { LD : 0,01 } \\
- \text { LQ : ND }\end{array}$ & $\begin{array}{l}\text { - Pratique de manipulation et exposition } \\
\text { non décrites } \\
\text { - Type : collecte urinaire de } 24 \mathrm{~h} \\
\text { - Période : février } 2006 \\
\text { - } 62 \text { prélèvements }\end{array}$ & $\begin{array}{l}\text { Exposés : } \\
11 / 62(18 \%) \\
\text { - Pharmaciens } \\
0 \% \\
\text { - Infirmiers ND } \\
\text { - Médecins ND }\end{array}$ & $\begin{array}{l}\text { Exposés : } \\
3 / 10(30 \%) \\
\text { - Pharmaciens } \\
0 / 3(0 \%) \\
\text { - Infirmiers } \\
\text { 2/4 (50\%) } \\
\text { - Médecins } \\
1 / 3(33 \%)\end{array}$ & $\begin{array}{l}\text { Exposés } \\
\text { (ng/24 h) } \\
\text { Min. : } 12 \\
\text { Max. : } 42 \\
\text { - Pharmaciens } \\
\text { < LD } \\
\text { - Infirmiers } \\
\text { (ng/24 h) } 34 \\
\text { et } 42 \\
\text { - Médecins } \\
\text { (ng/24 h) } 12\end{array}$ \\
\hline $\begin{array}{l}\text { Villarini et al. } \\
(2011)^{32} \\
\text { Italie }\end{array}$ & $\begin{array}{l}\text { Monocentrique (1) } \\
\text { Population } \\
\text { - Exposés (40) } \\
\text { Professions } \\
\text { - ATP (ND) } \\
\text { - Infirmiers (ND) } \\
\text { - Autres (ND) }\end{array}$ & $\begin{array}{l}\text { GC-MS } \\
C P \\
-L D: 0,1 \\
-L Q: N D\end{array}$ & $\begin{array}{l}\text { - Pratiques de manipulation et exposition } \\
\text { des } 6 \text { mois précédant la collecte décrites } \\
\text { grâce à un questionnaire } \\
\text { - Type : ponctuel } \\
\text { - Urines de fin de quart de travail } \\
\text { - Période : ND } \\
\text { - ND }\end{array}$ & ND & $\begin{array}{l}\text { Exposés: } \\
7 / 40(18 \%) \\
\text { (soit } 7 \\
\text { infirmiers) }\end{array}$ & $\begin{array}{l}\text { Exposés : } \\
\text { Min. : LD } \\
\text { Max. : 1,2 }\end{array}$ \\
\hline $\begin{array}{l}\text { Konate et al. } \\
(2011)^{34} \\
\text { France }\end{array}$ & $\begin{array}{l}\text { Monocentrique (1) } \\
\text { Population } \\
\text { - Exposés (11) } \\
\text { - Témoins (6) } \\
\text { Professions } \\
\text { - Infirmiers (ND) } \\
\text { - Médecins (ND) } \\
\text { - Autres (ND) }\end{array}$ & $\begin{array}{l}\text { ICP-MS } \\
\text { Platines } \\
\text { - LD : } 1,5 \times 10^{-3} \\
\text { - LQ : } 5 \times 10^{-3}\end{array}$ & $\begin{array}{l}\text { - Pratiques de manipulation non décrites. } \\
\text { Exposition décrite pour la procédure CHIP } \\
\text { - Type : ponctuel et répété } \\
\text { - } 1 \text { échantillon avant la CHIP } \\
\text { - } 1 \text { échantillon après la CHIP } \\
\text { - Ponctuel pour témoins } \\
\text { - Période : } 1 \text { jour } \\
\text { - } 24 \text { prélèvements }\end{array}$ & $\begin{array}{l}\text { Exposés avant } \\
\text { procédure : } \\
5 / 11(46 \%) \\
\text { Exposés après } \\
\text { procédure : } \\
3 / 7(43 \%) \\
\text { Témoins avant } \\
\text { procédure : } \\
1 / 6(17 \%)\end{array}$ & $\begin{array}{l}\text { Exposés avant } \\
\text { procédure : } \\
5 / 11(46 \%) \\
\text { Exposés après } \\
\text { procédure: } \\
3 / 7(43 \%) \\
\text { Témoins avant } \\
\text { procédure : } \\
1 / 6(17 \%)\end{array}$ & $\begin{array}{l}\text { Exposés avant } \\
\text { procédure : } \\
\text { Min. : LD } \\
\text { Max. : LQ } \\
\text { Exposés après } \\
\text { procédure : } \\
\text { Min. : LD } \\
\text { Max. : LQ } \\
\text { Témoins avant } \\
\text { procédure : } \\
5,8 \times 10^{-3}\end{array}$ \\
\hline $\begin{array}{l}\text { Sugiura et al. } \\
(2011)^{39} \\
\text { Japon }\end{array}$ & $\begin{array}{l}\text { Multicentrique (6) } \\
\text { Population } \\
\text { - Exposés (41) } \\
\text { Professions } \\
\text { - Infirmiers (13) } \\
\text { - Médecins (1) } \\
\text { - Pharmaciens (27) }\end{array}$ & $\begin{array}{l}\text { GC-MS/MS } \\
\text { CP } \\
\text { - LD : ND } \\
\text { - LQ : ND } \\
\text { 7) }\end{array}$ & $\begin{array}{l}\text { - Pratiques de manipulation et exposition } \\
\text { non décrites } \\
\text { - Type : collecte urinaire de } 24 \mathrm{~h} \\
\text { - Période : ND } \\
\text { - } 276 \text { prélèvements }\end{array}$ & $\begin{array}{l}\text { Exposés : } \\
\text { 90/276 (33 \%) } \\
\text { - Infirmiers : } \\
\text { 11/80 (14\%) } \\
\text { - Médecins : } \\
\text { 0/3 (0 \%) } \\
\text { - Pharmaciens : } \\
\text { 79/193 (41\%) }\end{array}$ & $\begin{array}{l}\text { Exposés: } \\
\text { 23/41 (56 \%) } \\
\text { - Infirmiers : } \\
\text { 5/13 (38\%) } \\
\text { - Médecins : } \\
\text { 0/1 (0 \%) } \\
\text { - Pharmaciens : } \\
\text { 18/27 (67 \%) }\end{array}$ & $\begin{array}{l}\text { Exposés } \\
\text { (ng/24 h) } \\
\text { Min. : 2,7 } \\
\text { Max. : 462,8 } \\
\text { - Infirmiers } \\
\text { (ng/24 h) } \\
\text { : Min. : 2,7 } \\
\text { Max. : 128,6 } \\
\text { - Médecins < LD } \\
\text { - Pharmaciens } \\
\text { (ng/24 h) } \\
\text { Min. : 3,2 } \\
\text { Max. : 462,8 }\end{array}$ \\
\hline
\end{tabular}


Tableau 1 (partie 6 de 7). Profil des résultats de 24 études de surveillance urinaire ${ }^{18-41}$ menées sur des professionnels de la santé exposés aux antinéoplasiques et publiées entre le 1"r janvier 2010 et le 31 décembre 2015*

\begin{tabular}{|c|c|c|c|c|c|c|}
\hline Étude et Pays & $\begin{array}{c}\text { Type } \\
\text { (n centres), } \\
\text { Population } \\
\text { (n travailleurs), } \\
\text { Professions } \\
\text { ( } n \text { travailleurs) } \\
\end{array}$ & $\begin{array}{l}\text { Méthode } \\
\text { lédicaments } \\
\text { - Limites } \\
\text { (ng/mL) }\end{array}$ & $\begin{array}{l}\text { Prélèvements urinaires } \\
\text { Pratiques de manipulation et exposition } \\
\text { - Type de collecte } \\
\text { - Période de collecte } \\
\text { - Prélèvements }(n)\end{array}$ & $\begin{array}{l}\text { Prélèvements } \\
\text { positifs } \\
n(\%)\end{array}$ & $\begin{array}{l}\text { Travailleurs } \\
\text { positifs } \\
n(\%)\end{array}$ & $\begin{array}{l}\text { Concentrations } \\
\text { (ng/mL) }\end{array}$ \\
\hline $\begin{array}{l}\text { Turci et al. } \\
(2011)^{40} \\
\text { Italie }\end{array}$ & $\begin{array}{l}\text { Multicentrique (7) } \\
\text { Population } \\
\text { - Exposés (102) } \\
\text { Professions } \\
\text { - Médecins (3) } \\
\text { - Autres (99-38 } \\
\text { à la préparation, } \\
61 \text { à } \\
\text { l'administration) }\end{array}$ & $\begin{array}{l}\text { HPLC-MS/MS } \\
\text { - CP, paclitaxel, } \\
\text { doxorubicine, } \\
\text { épirubicine } \\
\text { ICP-MS } \\
\text { - Platines } \\
\text { HPLC-UV } \\
\text {-5-FU } \\
\text { GC-MS } \\
\text { - FBAL } \\
\text { LQ : variables } \\
\text { LD : ND }\end{array}$ & $\begin{array}{l}\text { - Pratiques de manipulation et exposition } \\
\text { décrites et recueillies grâce à un } \\
\text { questionnaire et un entretien } \\
\text { - Type : ponctuel } \\
\text { - Urines avant le quart de travail } \\
\text { - Urines après le quart de travail } \\
\text { - } 1 \text { un échantillon de milieu de quart } \\
\text { - Période : } 2003-2007 \\
\text { - } 207 \text { prélèvements }\end{array}$ & $\begin{array}{l}\text { Exposés : } \\
\text { 0/207 (0 \%) }\end{array}$ & $\begin{array}{l}\text { Exposés : } \\
0 / 102(0 \%)\end{array}$ & Exposés : < LQ \\
\hline $\begin{array}{l}\text { Yoshida et al. } \\
(2011)^{41} \\
\text { Japon }\end{array}$ & $\begin{array}{l}\text { Multicentrique (5) } \\
\text { Population } \\
\text { • Exposés (17) } \\
\text { Professions } \\
\text { - Pharmaciens (17) }\end{array}$ & $\begin{array}{l}\text { GC-MS } \\
\text { CP } \\
\text { - Limite de } \\
\text { mesure : } \\
0,1 \mathrm{ng} \\
\text { ICP-MS } \\
\text { Platines } \\
\text { - Limite de } \\
\text { mesure: } \\
\text { 2,0 ng }\end{array}$ & $\begin{array}{l}\text { - Pratiques de manipulation et exposition } \\
\text { décrites et recueillies grâce à un entretien } \\
\text { - Type : collecte urinaire de } 24 \text { h } \\
\text { - Du début de la préparation jusqu'au } \\
\text { lendemain } \\
\text { - Période : septembre } 2007 \text { à mars } 2009 \\
\text { - ND }\end{array}$ & ND & $\begin{array}{l}\text { Exposés : } \\
-C P: 3 / 17 \\
(18 \%) \\
\text { - Platines : } \\
0 / 17(0 \%)\end{array}$ & $\begin{array}{l}\text { Exposés : } \\
- \text { CP }(\text { ng/24 h) } \\
3 \text { collectes } \\
\text { positives, soit } \\
6,7 ; 11 ; 52 \\
\text { - Platines : < LD }\end{array}$ \\
\hline $\begin{array}{l}\text { Connor et al. } \\
(2010)^{33} \\
\text { États-Unis }\end{array}$ & $\begin{array}{l}\text { Multicentrique (3) } \\
\text { Population } \\
\text { - Exposés (68) } \\
\text { - Témoins (53) } \\
\text { Professions (exposés } \\
\text { c. témoins) } \\
\text { - AS (4 c.6) } \\
\text { - ATP (8 c. } 2 \text { ) } \\
\text { - Infirmiers (47 c.33) } \\
\text { - Pharmaciens } \\
\text { (9 c.12) }\end{array}$ & $\begin{array}{l}\text { HPLC-MS/MS } \\
\text { CP et paclitaxel } \\
\text { - LD : 0,015 } \\
\text { - LQ : ND } \\
\text { 3) }\end{array}$ & $\begin{array}{l}\text { - Pratiques de manipulation et exposition } \\
\text { décrites avec un journal de bord quotidien } \\
\text { sur } 6 \text { semaines avant la collecte } \\
\text { - Type : collecte urinaire de } 2 \text { fois } 4 \text { heures } \\
\text { - } 4 \text { dernières heures du quart de travail } \\
\text { - } 4 \text { premières heures suivant la fin du quart } \\
\text { de travail } \\
\text { - Période : ND } \\
\text { - } 119 \text { prélèvements }\end{array}$ & $\begin{array}{l}\text { Exposés : } \\
3 / 67(5 \%) \\
\text { Témoins : } \\
0 / 52 \text { (0 \%) }\end{array}$ & $\begin{array}{l}\text { Exposés : } \\
3 / 67(5 \%) \\
\text { Témoins : } \\
0 / 52(0 \%)\end{array}$ & $\begin{array}{l}\text { Exposés : } \\
\text { CP }(2 \\
\text { pharmaciens }) \\
-0,043 \times 10^{-3} \\
-0,079 \times 10^{-3} \\
\text { paclitaxel } \\
(1 \text { ATP }): \\
-0,01 \times 10^{-3} \\
\text { Témoins : }<\text { LD }\end{array}$ \\
\hline $\begin{array}{l}\text { Maeda et al. } \\
(2010)^{35} \\
\text { Japon }\end{array}$ & $\begin{array}{l}\text { Monocentrique (1) } \\
\text { Population } \\
\text { - Exposés (8) } \\
\text { Professions } \\
\text { - Infirmiers (2) } \\
\text { - Pharmaciens (6) }\end{array}$ & $\begin{array}{l}\text { LC-MS/MS } \\
\text { CP et } \\
\text { ifosfamide } \\
\text { - LID : 0,4 } \\
\text { - LIQ : 0,4 }\end{array}$ & $\begin{array}{l}\text { - Pratiques de manipulation et exposition } \\
\text { avant la collecte urinaire décrites } \\
\text { - Type : échantillons ponctuels et répétés } \\
\text { - Échantillon durant le quart de travail } \\
\text { après administration du CP ( } 2 \text { infirmiers) } \\
\text { - Échantillon 6-10 h ou 20-24 h après } \\
\text { préparation de CP (6 pharmaciens) } \\
\text { - Type : collecte urinaire de } 24 \text { h } \\
\text { - Après préparation de CP (1 pharmacien) } \\
\text { - Période : ND } \\
\text { - } 35 \text { prélèvements }\end{array}$ & $\begin{array}{l}\text { Exposés : } \\
\text { 0/35 (0 \%) }\end{array}$ & $\begin{array}{l}\text { Exposés : } \\
0 / 8(0 \%)\end{array}$ & Exposés : < LID \\
\hline
\end{tabular}


This single copy is for your personal, non-commercial use only.

For permission to reprint multiple copies or to order presentation-ready copies for distribution, contact CJHP at cjhpedit@cshp.ca

\section{Tableau 1 (partie 7 de 7). Profil des résultats de 24 études de surveillance urinaire ${ }^{18-41}$ menées sur des professionnels de la santé exposés aux antinéoplasiques et publiées entre le 1er janvier 2010 et le 31 décembre 2015*}

\begin{tabular}{|c|c|c|c|c|c|c|}
\hline Étude et Pays & $\begin{array}{c}\text { Type } \\
\text { (n centres), } \\
\text { Population } \\
\text { (n travailleurs), } \\
\text { Professions } \\
\text { (n travailleurs) } \\
\end{array}$ & $\begin{array}{l}\text { Méthode } \\
\text { Médicaments } \\
\text { - Limites } \\
\text { (ng/mL) }\end{array}$ & $\begin{array}{l}\text { Prélèvements urinaires } \\
\text { Pratiques de manipulation et exposition } \\
\text { - Type de collecte } \\
\text { - Période de collecte } \\
\text { - Prélèvements }(n)\end{array}$ & $\begin{array}{c}\text { Prélèvements } \\
\text { positifs } \\
n(\%)\end{array}$ & $\begin{array}{c}\text { Travailleurs } \\
\text { positifs } \\
n(\%)\end{array}$ & $\begin{array}{l}\text { Concentrations } \\
(\mathrm{ng} / \mathrm{mL})\end{array}$ \\
\hline $\begin{array}{l}\text { Näslund } \\
\text { Andréasson } \\
\text { et al. }(2010)^{36} \\
\text { Suède }\end{array}$ & $\begin{array}{l}\text { Monocentrique (1) } \\
\text { Population } \\
\text { - Exposés (2) } \\
\text { Professions } \\
\text { - Médecin (1) } \\
\text { - Autre (1) }\end{array}$ & $\begin{array}{l}\text { ICP-SFMS } \\
\text { Platines } \\
\text { (oxaliplatine) } \\
\text { - LD: } 0,01 \\
\text { - LQ : ND }\end{array}$ & $\begin{array}{l}\text { - Pratiques de manipulation et exposition } \\
\text { de chaque procédure CHIP décrites } \\
\text { - Type : ponctuel et répété } \\
\text { - Urine avant l'intervention chirurgicale } \\
\text { (CHIP) } \\
\text { - Urine } 2 \text { h après la fin du traitement } \\
\text { - Urine } 12 \text { à } 15 \text { h après l'intervention } \\
\text { chirurgicale (CHIP) } \\
\text { - Période : } 2008 \\
\text { - } 36 \text { prélèvements }\end{array}$ & $\begin{array}{l}\text { Exposés : } \\
0 / 36(0 \%)\end{array}$ & $\begin{array}{l}\text { Exposés : } \\
0 / 2(0 \%)\end{array}$ & $\begin{array}{l}\text { Exposés: } \\
<\mathrm{LD}\end{array}$ \\
\hline $\begin{array}{l}\text { Ndaw et al. } \\
(2010)^{37} \\
\text { France }\end{array}$ & $\begin{array}{l}\text { Monocentrique (1) } \\
\text { Population } \\
\text { - Exposés (19) } \\
\text { Professions } \\
\text { - AS (8) } \\
\text { - ATP (6) } \\
\text { - Infirmiers (5) }\end{array}$ & $\begin{array}{l}\text { HPLC-MS/MS } \\
\text { FBAL } \\
- \text { LD : ND } \\
- \text { LQ : } 1\end{array}$ & $\begin{array}{l}\text { - Pratiques de manipulation et exposition } \\
\text { non décrites } \\
\text { - Type : ponctuel et répété } \\
\text { - } 1 \text { échantillon en fin de quart de travail } \\
\text { (64 prélèvements) } \\
\text { - } 1 \text { échantillon après le quart de travail } \\
\text { (57 prélèvements) } \\
\text { - Période : } 5 \text { jours consécutifs } \\
\text { - } 121 \text { prélèvements }\end{array}$ & $\begin{array}{l}\text { Exposés : } \\
35 / 121(29 \%) \\
\text { - AS : } 16 / 43 \\
\text { (37\%) } \\
\text { - ATP : } 15 / 52 \\
\text { (29\%) } \\
\text { - Infirmiers : } \\
\text { 4/26 (15\%) }\end{array}$ & $\begin{array}{l}\text { Exposés : } \\
14 / 19(74 \%) \\
\text { - AS : } \\
\text { 7/8 (88 \%) } \\
\text { - ATP : } \\
\text { 5/6 (83 \%) } \\
\text { - Infirmiers : } \\
\text { 2/5 (40\%) }\end{array}$ & $\begin{array}{l}\text { Exposés : } \\
\text { Min. : LQ } \\
\text { Max. : } 22,7 \\
\text { - AS : } \\
\text { Min. : 1,00 } \\
\text { Max. : 9,85 } \\
\text { - ATP : } \\
\text { Min. : 1,17 } \\
\text { Max. : 6,06 } \\
\text { - Infirmiers : } \\
\text { Min. : 1,27 } \\
\text { Max. : 22,7 }\end{array}$ \\
\hline $\begin{array}{l}\text { Pieri et al. } \\
(2010)^{38} \\
\text { Italie }\end{array}$ & $\begin{array}{l}\text { Multicentrique (2) } \\
\text { Population } \\
\text { - Exposés (56) } \\
\text { Professions } \\
\text { - Infirmiers (56) }\end{array}$ & $\begin{array}{l}\text { HPLC-FL } \\
\text { Doxorubicine } \\
\text { - LD : } 0,6 \\
\text { - LIQ : } 1,1 \\
\text { Épirubicine } \\
\text { - LD : } 1,2 \\
\text { - LIQ : } 2,0\end{array}$ & $\begin{array}{l}\text { - Pratiques de manipulation et exposition } \\
\text { décrites et recueillies grâce à un } \\
\text { questionnaire } \\
\text { - Type : ponctuel } \\
\text { - } 1 \text { échantillon en fin de quart de travail } \\
\text { - Période : ND } \\
\text { - } 56 \text { prélèvements }\end{array}$ & $\begin{array}{l}\text { Exposés : } \\
5 / 56(9 \%)\end{array}$ & $\begin{array}{l}\text { Exposés : } \\
5 / 56(9 \%)\end{array}$ & $\begin{array}{l}\text { Exposés : } \\
\text { Doxorubicine : } \\
17,0 \text { et } 33,9 \\
\text { Épirubicine : } \\
42,0 ; 60,7 ; \\
72,6 ; 84,1\end{array}$ \\
\hline
\end{tabular}

$\mathrm{AP}=$ aides-pharmaciens, $\mathrm{AS}=$ aides-soignants, $\mathrm{ATP}=$ assistants techniques en pharmacie, $C H I P=$ chimiothérapie hyperthermique intrapéritonéale, com. = communautaire, $\mathrm{CP}=$ cyclophosphamide, FBAL = fluoro-béta-alanine, GC-MS = gas chromatography-mass spectrometry (chromatographie en phase gazeuse couplée à la spectrométrie de masse), GC-MS/MS : gas chromatography-tandem mass spectrometry (chromatographie en phase gazeuse couplée à la spectrométrie de masse en tandem), HPLC-FL = high-performance liquid chromatography-fluorescence (chromatographie en phase liquide à haute performance couplée à la détection en fluorescence), HPLC-MS/MS = high-performance liquid chromatography-tandem mass spectrometry (chromatographie en phase liquide à haute performance couplée à la spectrométrie de masse en tandem),

HPLC-UV = high-performance liquid chromatography interfaced with an ultraviolet detector (chromatographie en phase liquide à haute performance interfacée à un détecteur ultraviolet), ICP-MS = inductively coupled plasma mass spectrometry (spectrométrie de masse à plasma à couplage inductif), ICP-SFMS = inductively coupled plasma-sector field mass spectrometry (spectrométrie de masse à plasma à couplage inductif à secteur magnétique), LC-MS/MS = liquid chromatography-tandem mass spectrometry (chromatographie en phase liquide couplée à la spectrométrie de masse en tandem), LD = limite de détection, LID = limite inférieure de détection, LIQ = limite inférieure de quantification, $\mathrm{LQ}=$ limite de quantification, $\mathrm{ND}=$ non disponible.

* Les études sont présentées en ordre décroissant d'année de publication.

celle de deux ou de plusieurs médicaments. Les prélèvements urinaires provenaient de collectes ponctuelles $(n=12)$, de collectes sur une période de 24 heures $(n=10)$, sur une période de 29 heures $(n=1)$, sur une période de 28 à 30 heures $(n=1)$, sur une période de 8 heures $(n=1)$ et sur une période de 4 heures $(n=1)$.

L'ensemble des résultats de six études, qui portaient sur 155 travailleurs, étaient négatifs, dont trois ayant procédé à des collectes ponctuelles et répétées, deux à des collectes ponctuelles et une à une collecte sur une période de 24 heures. Notons que cinq des six études comportaient au moins un prélèvement de surface positif réalisé parallèlement à des mesures urinaires. Seize autres études comportaient des résultats positifs. Enfin, deux autres études démontraient l'utilité de mesures répétées. L'une a démontré que le pourcentage de travailleurs dont les échantillons étaient positifs au cyclophosphamide ou au méthotrexate lors de mesures répétées entre 2001 et 2010 avait diminué de $36 \%$ à $0 \%{ }^{27}$. L'autre a démontré que le taux d'échantillons positifs 
au cyclophosphamide avait passé de $45 \%$ à $0 \%$ après une sensibilisation du personnel au moyen d'une liste de vérification des pratiques ${ }^{28}$.

Une seule étude présentait une comparaison avant et après l'utilisation d'un système fermé pour la préparation et l'administration des médicaments dangereux. Elle a révélé que le pourcentage de travailleurs dont les échantillons étaient positifs avait diminué de $100 \%$ à $50 \%{ }^{25}$.

Au total, 1648 collectes urinaires ont été réalisées. Il y avait une grande variabilité entre les valeurs obtenues. Par exemple, les collectes sur une période de 24 heures ont démontré que la plus faible valeur de cyclophosphamide était de 2,7 ng/24 heures et la plus élevée, de $474 \mathrm{ng} / 24$ heures. Les prélèvements ponctuels ont révélé que la plus faible valeur positive du cyclophosphamide était de $0,043 \times 10^{-3} \mathrm{ng} / \mathrm{mL}$ et que la valeur la plus élevée était de $10 \mathrm{ng} / \mathrm{mL}$.

Cinq études se sont intéressées à la surveillance urinaire de professionnels de la santé lors de procédures spécifiques : quatre études concernaient la chimiothérapie hyperthermique intrapéritonéale (CHIP), et une portait sur un système de préparation robotique. Une seule étude traitant de la CHIP mentionnait une contamination urinaire des travailleurs.

Cinq articles ne donnaient aucune information sur les pratiques de manipulation et d'exposition, et deux décrivaient uniquement l'exposition.

La proportion de travailleurs exposés à des médicaments dangereux, dont le prélèvement d'urine était positif, s'étendait de $0 \%(n=10$ études) à $100 \%(n=4)$. Si on ne retient que les études permettant de calculer le taux de travailleurs déclarés positifs $(n=23)$, la proportion totale de travailleurs comportant au moins un prélèvement urinaire positif était de $21 \%$ (173/809), toutes molécules et méthodes analytiques confondues. Il faut noter que, lorsque plusieurs situations étaient mises en comparaison, ce calcul ne prenait en compte que la situation la plus risquée (p. ex. manipulation avant la mise en place du système fermé, travailleur exposé après une procédure de $\mathrm{CHIP}$ ).

Dans les 11 études portant sur le cyclophosphamide, la proportion totale de travailleurs ayant produit au moins un prélèvement urinaire positif était de 38 \% (103/270). Dans les six études portant sur les platines, la proportion totale de travailleurs était de $19 \%(13 / 70)$.

\section{DISCUSSION}

\section{Professionnels de la santé exposés aux médicaments dangereux}

Cette revue documentaire a mis en évidence 24 études publiées sur la contamination urinaire de professionnels de la santé au sein de sept pays. Ces études ne représentent que 826 travailleurs exposés à des médicaments dangereux dans le monde. Il s'agit d'un nombre très limité si l'on considère que 1,2 millions de personnes dispensent des soins de santé au Canada, dont plus de 365000 infirmières, plus de
75000 médecins et plus de 33400 pharmaciens $^{42}$. Ces chiffres canadiens ne tiennent pas compte du nombre d'assistants techniques en pharmacie. Tous les dispensateurs de soins ne sont pas forcément exposés à des médicaments dangereux, mais considérant le nombre croissant de médicaments classés comme dangereux par le NIOSH, l'exposition professionnelle est une préoccupation qui dépasse les frontières de l'oncologie et de l'hôpital.

\section{Diversité des études}

Il existe plusieurs méthodes analytiques différentes permettant la mesure urinaire de 13 médicaments dangereux utilisés en oncologie. De plus, il est surprenant de constater que certains articles ne décrivent pas les pratiques de manipulation et l'exposition réelle des travailleurs qui se sont révélées positives ou non aux témoins urinaires. Les descriptions sont tellement diversifiées que la comparaison entre les études s’avère difficile.

\section{Réduction de la contamination}

Six études n'ont pas détecté de traces urinaires chez les 155 travailleurs exposés aux médicaments dangereux. Il est difficile d'établir le type d'intervention ayant contribué à réduire ou à négativer les traces de médicaments dangereux dans l'urine des professionnels de la santé. Plusieurs auteurs évoquent le respect accru des mesures de protection.

Deux études ont comparé les taux de contamination de ses travailleurs au fil des ans et ont constaté une diminution de la contamination. Nous croyons donc que le fait d'augmenter le degré de conscience des risques et de connaissance des mesures de protection permet de réduire la contamination. En effet, une de ces études avait implanté un score de qualité entre les deux moments de mesure. Une autre étude a démontré une diminution de la contamination à la suite de l'implantation d'un circuit fermé; néanmoins, plusieurs mois se sont écoulés entre les deux mesures, donc un effet d'amélioration lié au temps a pu contribuer à cette diminution.

Il est encourageant de constater que plusieurs études n'ont pas trouvé de contaminations urinaires dans l'urine des travailleurs. Ainsi, les établissements de santé appliquant les bonnes pratiques de travail, ayant accès à des équipements sécuritaires et fournissant des équipements de protection appropriés peuvent limiter la contamination des professionnels de la santé.

\section{Pertinence d'un programme de surveillance}

Les articles recensés entre 2010 et 2015 représentent des études effectuées dans un cadre de recherche. En dépit de toutes les études publiées, il n'existe actuellement pas suffisamment de balises juridiques permettant de soutenir la mise en place d'un programme provincial ou national de surveillance urinaire. Certains points demeurent à clarifier, notamment sur le plan de l'assurabilité des travailleurs dont l'analyse des prélèvements serait 
positive et des possibilités de poursuites judiciaires. Nous pensons que la divulgation individuelle des résultats aux travailleurs concernés peut être envisagée uniquement après la tenue d'une première étude visant à caractériser l'état de situation.

De plus, les pratiques optimales de prélèvement sont variables selon le type de collecte et les médicaments à quantifier. Un programme idéal de surveillance comporterait aussi un bon rapport coût-avantage. Par exemple, la quantification d'un grand nombre de médicaments par collecte urinaire sur une période de 24 heures augmenterait les probabilités de détection, mais cette option serait trop coûteuse pour les régimes de soins de santé qui sont confrontés à une crise financière. En outre, la mise en place d'un programme de surveillance urinaire comporte plusieurs conditions de succès, notamment l'entérinement par un organisme en santé et sécurité au travail, le soutien local au sein de chaque établissement, l'information du personnel, l'inclusion des cliniciens dans une revue périodique des pratiques afin de soutenir les changements requis en présence de résultats positifs.

\section{Limites}

Cette revue de littérature scientifique ne cible que les études publiées de 2010 à 2015. Nous avons choisi de cibler notre recherche documentaire sur les cinq dernières années compte tenu de l'évolution des connaissances et des pratiques. Nous avons décidé d'intégrer toutes les études dans cette revue de littérature même s'il est difficile de comparer les études entre elles compte tenu de l'hétérogénéité des méthodes analytiques, des méthodes de prélèvements et de la présentation des résultats. De plus, chaque médicament présente une pharmacocinétique et une pharmacodynamie différentes. Chaque établissement de santé comporte un environnement physique distinct et des pratiques professionnelles variables. Le taux d'adhésion à ces pratiques varie considérablement dans le temps et entre les personnes. Ainsi, la diversité des méthodologies présentées ici peut permettre aux futures études d'anticiper toute la problématique de la surveillance urinaire et peut-être d'améliorer leur méthodologie. De plus, elle peut contribuer à l'interprétation des données d'autres études.

\section{CONCLUSION}

Cette revue de la littérature scientifique a mis en évidence 24 études de surveillance urinaire menées de 2010 à 2015 et portant sur des professionnels de la santé au sein de sept pays. Dans plusieurs études, aucune trace de médicaments antinéoplasiques n'a été trouvée dans les urines. Il serait souhaitable que les prochaines études décrivent précisément les pratiques de manipulation et l'exposition des professionnels de santé, car ces paramètres sont importants pour permettre la comparaison des études entre elles et l'identification des facteurs responsables de contamination. Les études publiées discutent majoritairement des professionnels de la santé qui manipulent directement les médicaments dangereux : infirmières, pharmaciens, assistants techniques, médecins. Toutefois, il serait intéressant d'évaluer la contamination des travailleurs exposés indirectement à des médicaments dangereux, tels que les commis, le personnel d'hygiène et de salubrité et le personnel de bureau.

\section{Références}

1. NIOSH Alert. Preventing occupational exposures to antineoplastic and other hazardous drugs in health care settings. Cincinnati $(\mathrm{OH})$ : Department of Health and Human Services (É.U.), Centers for Disease Control and Prevention, National Institute for Occupational Safety and Health; 2004. Publié au : www.cdc.gov/niosh/docs/2004-165/pdfs/2004-165.pdf. Consulté le 23 janvier 2016.

2. Connor TH, MacKenzie BA, DeBord DG, Trout DB, O'Callaghan JP. $\mathrm{NIOSH}$ list of antineoplastic and other hazardous drugs in healthcare settings, 2014. Cincinnati (OH): Department of Health and Human Services (É.U.), Centers for Disease Control and Prevention, National Institute for Occupational Safety and Health; 2014. Publié au : www.cdc.gov/ niosh/docs/2014-138/pdfs/2014-138.pdf. Consulté le 24 janvier 2016.

3. Guide de prévention - Manipulation sécuritaire des médicaments dangereux [GP65]. Montréal (QC) : Association paritaire pour la santé et la sécurité du travail du secteur affaires sociales; 2008. Publié au : www.asstsas.qc.ca/ publication/guide-de-prevention-manipulation-securitaire-des-medicamentsdangereux-gp65. Consulté le 28 janvier 2016.

4. Préparations de produits stériles dangereux en pharmacie. Norme 2014.02. Montréal (QC) : Ordre des pharmaciens du Québec; 2014. Publié au : www.opq.org/cms/Media/1847_38_fr-CA_0_Norme_2014_02.pdf. Consulté le 27 janvier 2016.

5. Medical surveillance for healthcare workers exposed to hazardous drugs. Cincinnati $(\mathrm{OH})$ : National Institute for Occupational Safety and Health (É.U.); 2012. Publié au : www.cdc.gov/niosh/docs/wp-solutions/2013103/pdfs/ 2013-103.pdf. Consulté le 28 janvier 2016.

6. Connor TH, Lawson CC, Polovich M, McDiarmid MA. Reproductive health risks associated with occupational exposures to antineoplastic drugs in health care settings: a review of the evidence. J Occup Environ Med. 2014;56(9):901-10.

7. El-Ebiary AA, Abuelfadl AA, Sarhan NI. Evaluation of genotoxicity induced by exposure to antineoplastic drugs in lymphocytes of oncology nurses and pharmacists. J Appl Toxicol. 2013;33(3):196-201.

8. Connor TH, Anderson RW, Sessink PJ, Broadfield L, Power LA. Surface contamination with antineoplastic agents in six cancer treatment centers in Canada and the United States. Am J Health Syst Pharm. 1999;56(14):1427-32.

9. Touzin K, Bussières JF, Langlois E, Lefebvre M. Evaluation of surface contamination in a hospital hematology-oncology pharmacy. J Oncol Pharm Pract. 2009;15(1):53-61.

10. Bussières JF, Tanguay C, Touzin K, Langlois É, Lefebvre M. Environmental contamination with hazardous drugs in Quebec hospitals. Can J Hosp Pharm. 2012;65(6):428-35.

11. Merger D, Tanguay C, Langlois É, Lefebvre M, Bussières JF. Environmental contamination with methotrexate in Canadian community pharmacies. J Am Pharm Assoc. 2013;53(4):423-6.

12. Janes A, Tanguay C, Caron NJ, Bussières JF. Environmental contamination with cyclophosphamide, ifosfamide, and methotrexate: a study of 51 Canadian centres. Can J Hosp Pharm. 2015;68(4):279-89.

13. Berruyer M, Tanguay C, Caron NJ, Lefebvre M, Bussières JF. Multicenter study of environmental contamination with antineoplastic drugs in 36 Canadian hospitals: a 2013 follow-up study. J Occup Environ Hyg. 2015; 12(2):87-94.

14. Merger D, Tanguay C, Langlois E, Lefebvre M, Bussières JF. Multicenter study of environmental contamination with antineoplastic drugs in 33 Canadian hospitals. Int Arch Occup Environ Health. 2014;87(3):307-13.

15. Hon CY, Teschke K, Demers PA, Venners S. Antineoplastic drug contamination on the hands of employees working throughout the hospital medication system. Ann Occup Hyg. 2014;58(6):761-70.

16. Hon CY, Astrakianakis G, Danyluk Q, Chu W. Pilot evaluation of dermal contamination by antineoplastic drugs among hospital pharmacy personnel. Can J Hosp Pharm. 2011;64(5):327-32. 
17. Chu WC, Hon CY, Danyluk Q, Chua PP, Astrakianakis G. Pilot assessment of the antineoplastic drug contamination levels in British Columbian hospitals pre- and post-cleaning. J Oncol Pharm Pract. 2012;18(1):46-51.

18. Friese CR, McArdle C, Zhao T, Sun D, Spasojevic I, Polovich M, et al. Antineoplastic drug exposure in an ambulatory setting: a pilot study. Cancer Nurs. 2015;38(2):111-7.

19. Hon CY, Teschke K, Shen H, Demers PA, Venners S. Antineoplastic drug contamination in the urine of Canadian healthcare workers. Int Arch Occup Environ Health. 2015;88(7):933-41.

20. Ramphal R, Bains T, Goulet G, Vaillancourt R. Occupational exposure to chemotherapy of pharmacy personnel at a single centre. Can J Hosp Pharm. 2015;68(2):104-12.

21. Sessink PJ, Leclercq GM, Wouters DM, Halbardier L, Hammad C, Kassoul N. Environmental contamination, product contamination and workers exposure using a robotic system for antineoplastic drug preparation. J Oncol Pharm Pract. 2015;21(2):118-27.

22. Villa AF, El Balkhi S, Aboura R, Sageot H, Hasni-Pichard H, Pocard M, et al. Evaluation of oxaliplatin exposure of healthcare workers during heated intraperitoneal perioperative chemotherapy (HIPEC). Ind Health. 2015; 53(1):28-37.

23. Caneparo A, Massucco P, Vaira M, Maina G, Giovale E, Coggiola M, et al. Contamination risk for operators performing semi-closed HIPEC procedure using cisplatin. Eur J Surg Oncol. 2014;40(8):925-9.

24. Ramphal R, Bains T, Vaillancourt R, Osmond MH, Barrowman N. Occupational exposure to cyclophosphamide in nurses at a single center. J Occup Environ Med. 2014;56(3):304-12.

25. Miyake T, Iwamoto T, Tanimura M, Okuda M. Impact of closed-system drug transfer device on exposure of environment and healthcare provider to cyclophosphamide in Japanese hospital. Springerplus. 2013;2(1):273.

26. Kopp B, Crauste-Manciet S, Guibert A, Mourier W, Guerrault-Moro MN, Ferrari S, et al. Environmental and biological monitoring of platinumcontaining drugs in two hospital pharmacies using positive air pressure isolators. Ann Occup Hyg. 2013;57(3):374-83.

27. Sabatini L, Barbieri A, Lodi V, Violante FS. Biological monitoring of occupational exposure to antineoplastic drugs in hospital settings. Med Lav. 2012;103(5):394-401.

28. Yoshida J, Koda S, Nishida S, Nakano H, Tei G, Kumagai S. Association between occupational exposure and control measures for antineoplastic drugs in a pharmacy of a hospital. Ann Occup Hyg. 2013;57(2):251-60.

29. Sottani C, Porro B, Imbriani M, Minoia C. Occupational exposure to antineoplastic drugs in four Italian health care settings. Toxicol Lett. 2012; 213(1):107-15.

30. Hama K, Fukushima K, Hirabatake M, Hashida T, Kataoka K. Verification of surface contamination of Japanese cyclophosphamide vials and an example of exposure by handling. J Oncol Pharm Pract. 2012;18(2):201-6.

31. Sugiura S, Asano M, Kinoshita K, Tanimura M, Nabeshima T. Risks to health professionals from hazardous drugs in Japan: a pilot study of environmental and biological monitoring of ocupational exposure to cyclophosphamide. $J$ Oncol Pharm Pract. 2011;17(1):14-9.

32. Villarini M, Dominici L, Piccinini R, Fatigoni C, Ambrogi M, Curti G, et al. Assessment of primary, oxidative and excision repaired DNA damage in hospital personnel handling antineoplastic drugs. Mutagenesis. 2011; 26(3):359-69.

33. Connor TH, DeBord DG, Pretty JR, Oliver MS, Roth TS, Lees PS, et al. Evaluation of antineoplastic drug exposure of health care workers at three university-based US cancer centers. J Occup Environ Med. 2010; 52(10):1019-27.

34. Konate A, Poupon J, Villa A, Garnier R, Hasi-Pichard H, Mezzaroba D, et al. Evaluation of environmental contamination by platinum and exposure risk for healthcare workers during a heated intraperitoneal perioperative chemotherapy (HIPEC) procedure. J Surg Oncol. 2011;103(1):6-9.

35. Maeda S, Miyawaki K, Matsumoto S, Oishi M, Miwa Y, Kurokawa N. Evaluation of environmental contaminations and occupational exposures involved in preparation of chemotherapeutic drugs. Yakugaku Zasshi. 2010;130(6):903-10.
36. Näslund Andréasson S, Anundi H, Thorén SB, Ehrsson H, Mahteme H. Is platinum present in blood and urine from treatment givers during hyperthermic intraperitoneal chemotherapy? J Oncol. 2010;2010:649719.

37. Ndaw S, Denis F, Marsan P, d'Almeida A, Robert A. Biological monitoring of occupational exposure to 5-fluorouracil: urinary $\alpha$-fluoro- $\beta$-alanine assay by high performance liquid chromatography tandem mass spectrometry in health care personnel. J Chromatogr B Analyt Technol Biomed Life Sci. 2010; 878(27):2630-4

38. Pieri M, Castiglia L, Basilicata P, Sannolo N, Acampora A, Miraglia N. Biological monitoring of nurses exposed to doxorubicin and epirubicin by a validated liquid chromatography/fluorescence detection method. Ann Occup Hyg. 2010;54(4):368-76.

39. Sugiura $S$, Nakanishi $H$, Asano M, Hashida T, Tanimura M, Hama T, et al. Multicenter study for environmental and biological monitoring of occupational exposure to cyclophosphamide in Japan. J Oncol Pharm Pract. 2011;17(1):20-8.

40. Turci R, Minoia C, Sottani C, Coghi R, Severi P, Castriotta C, et al. Occupational exposure to antineoplastic drugs in seven Italian hospitals: the effect of quality assurance and adherence to guidelines.J Oncol Pharm Pract. 2011;17(4):320-32

41. Yoshida J, Koda S, Nishida S, Yoshida T, Miyajima K, Kumagai S. Association between occupational exposure levels of antineoplastic drugs and work environment in five hospitals in Japan. J Oncol Pharm Pract. 2011;17(1):29-38.

42. Les dispensateurs de soins de santé au Canada: profils provinciaux de 2012. Ottawa $(\mathrm{ON})$ : Institut canadien d'information sur la santé; 2014. Publié au : https://secure.cihi.ca/estore/productFamily.htm?locale=fr\&pf=PFC2500. Consulté le 23 janvier 2016.

Céline Poupeau est une assistante de recherche, Unité de recherche en pratique pharmaceutique, Centre hospitalier universitaire Sainte-Justine, Montréal, Québec. Elle est aussi une étudiante dans le programme D.Pharm. dans la Faculté des Sciences Pharmaceutiques et Biologiques de Nancy, Université de Nancy 1, Nancy, France.

Christel Roland est une assistante de recherche, Unité de recherche en pratique pharmaceutique, Centre hospitalier universitaire Sainte-Justine, Montréal, Québec. Elle est aussi une étudiante dans le programme D.Pharm. dans la Faculté des Sciences Pharmaceutiques et Biologiques de Lille, Université Lille 2 Droit et Santé, Lille, France.

Jean-François Bussières, B. Pharm., M. Sc., MBA, FCSHP, est chef, Unité de recherche en pratique pharmaceutique et Départment de pharmacie, Centre hospitalier universitaire Sainte-Justine, et professeur titulaire de clinique, Faculté de pharmacie, Université de Montréal, Montréal, Québec.

Intérêts concurrents : Aucun déclaré

\section{Adresse de correspondance :}

Jean-François Bussières

Département de pharmacie

Centre hospitalier universitaire Sainte-Justine

3175, chemin de la Côte Sainte-Catherine

Montréal QC H3T 1C5

Courriel : jf.bussieres@ssss.gouv.qc.ca

Financement : Aucun.

Remerciements : Les auteurs remercient Cynthia Tanguay, coordonnatrice, Unité de recherche en pratique pharmaceutique, Centre hospitalier universitaire Sainte-Justine, pour l'aide à l'analyse des données et la relecture du manuscrit. 